OPEN ACCESS

Edited by:

Jean-Yves Le Guennec, Université de Montpellier, France

Reviewed by:

Clemens Möller,

Hochschule Albstadt-Sigmaringen,

Germany

Timm Danker,

Natural and Medical Sciences

Institute, Germany

*Correspondence:

Arnaud Billet

arnaud.billet01@univ-poitiers.fr

Specialty section:

This article was submitted to

Pharmacology of lon Channels and Channelopathies, a section of the journal

Frontiers in Pharmacology

Received: 16 February 2017

Accepted: 24 March 2017

Published: 07 April 2017

Citation:

Billet A, Froux L, Hanrahan JW and Becq F (2017) Development of Automated Patch Clamp Technique to Investigate CFTR Chloride Channel

Function. Front. Pharmacol. 8:195 doi: 10.3389/fphar.2017.00195

\section{Development of Automated Patch Clamp Technique to Investigate CFTR Chloride Channel Function}

\author{
Arnaud Billet ${ }^{1 *}$, Lionel Froux ${ }^{1}$, John W. Hanrahan ${ }^{2,3,4}$ and Frederic Becq ${ }^{1}$ \\ ' Laboratoire Signalisation et Transports Ioniques Membranaires, Université de Poitiers - ERL7368, Centre National de la \\ Recherche Scientifique, Poitiers, France, ${ }^{2}$ Department of Physiology, McGill University, Montreal, QC, Canada, ${ }^{3}$ McGill \\ Cystic Fibrosis Translational Research Centre, Montreal, QC, Canada, ${ }^{4}$ The Research Institute of the McGill University Health \\ Centre, Montreal, QC, Canada
}

The chloride $\left(\mathrm{Cl}^{-}\right)$channel cystic fibrosis transmembrane conductance regulator (CFTR) is defective in cystic fibrosis (CF), and mutation of its encoding gene leads to various defects such as retention of the misfolded protein in the endoplasmic reticulum, reduced stability at the plasma membrane, abnormal channel gating with low open probability, and thermal instability, which leads to inactivation of the channel at physiological temperature. Pharmacotherapy is one major therapeutic approach in the CF field and needs sensible and fast tools to identify promising compounds. The high throughput screening assays available are often fast and sensible techniques but with lack of specificity. Few works used automated patch clamp (APC) for CFTR recording, and none have compared conventional and planar techniques and demonstrated their capabilities for different types of experiments. In this study, we evaluated the use of planar parallel APC technique for pharmacological search of CFTR-trafficking correctors and CFTR function modulators. Using optimized conditions, we recorded both wt- and corrected F508del-CFTR $\mathrm{Cl}^{-}$currents with automated whole-cell patch clamp and compared the data to results obtained with conventional manual whole-cell patch clamp. We found no significant difference in patch clamp parameters such as cell capacitance and series resistance between automated and manual patch clamp. Also, the results showed good similarities of CFTR currents recording between the two methods. We showed that similar stimulation protocols could be used in both manual and automatic techniques allowing precise control of temperature, classic IN relationship, and monitoring of current stability in time. In conclusion, parallel patch-clamp recording allows rapid and efficient investigation of CFTR currents with a variety of tests available and could be considered as new tool for medium throughput screening in CF pharmacotherapy.

Keywords: CFTR, automated patch clamp, medium throughput assay, physiological temperature recording, current stability

\section{INTRODUCTION}

The cystic fibrosis transmembrane conductance regulator (CFTR) is a cAMP-dependent chloride $\left(\mathrm{Cl}^{-}\right)$channel whose encoding gene is mutated in the common autosomal recessive disease cystic fibrosis (CF; Riordan et al., 1989). Mutations of the CFTR gene have been grouped into six classes according to their effects on synthesis, maturation, and function of the CFTR protein. The most 
common CFTR mutation is the deletion of the phenylalanine 508 residue (F508del-CFTR), a mutation present on at least one allele in $\sim 90 \%$ of patients with CF. The F508del mutation induces misfolding of the nascent protein and retention in the endoplasmic reticulum (Cheng et al., 1990), reduced stability at the plasma membrane (Lukacs et al., 1993), abnormal channel gating with low open probability (Dalemans et al., 1991), and thermal instability, which leads to inactivation of the channel at physiological temperature (Wang et al., 2011; Liu et al., 2012).

Cystic fibrosis transmembrane conductance regulator pharmacotherapy is one major therapeutic strategy that involves identifying compounds that correct abnormal CFTR protein. Most research effort is directed at finding small molecules which interact directly with mutated CFTR to correct its folding and gating, and thus improve protein trafficking and channel function. Two compounds that have yielded promising results are the potentiator Ivacaftor, also called VX770 (Van Goor et al., 2009), which was approved by the Food and Drug Administration (FDA) in 2012 as Kalydeco ${ }^{\mathrm{TM}}$ for patients over 12 years of age carrying the G551D mutation (5\% of patients), and the corrector Lumacaftor, also called VX809 (Van Goor et al., 2011). Lumacaftor was approved for clinical use in combination with Ivacaftor for the treatment of F508del homozygous patients; however, this combination therapy, called Orkambi ${ }^{\mathrm{TM}}$, provided only modest benefit (Boyle et al., 2014) indicating that more efficacious correctors are still needed. Therefore, the search for innovative small molecules and understanding their mechanisms of action remain a priority for CF therapeutic research. To this end, robust screening assays are essential for rapidly testing large numbers of compounds and performing informative concentration-response curves in vitro. As multiple CFTR defects need to be corrected, cell-based assays should monitor improvements in the trafficking, membrane stability, and channel function of rescued mutant CFTR.

One of the most powerful techniques for characterizing ion channels and studying their pharmacology is patch clamp recording. It allows the recording of channel currents in real time, in a biological environment, and with high resolution (Hamill et al., 1981). Unlike other functional assays such as Ussing chamber short-circuit current measurements, flux studies (using either radioisotopes or ion-selective electrodes), or fluorescence assays [membrane potential detection (Van Goor et al., 2006; Maitra et al., 2013) or yellow fluorescent protein (YFP) quenching (Galietta et al., 2001; Pedemonte et al., 2011)], patch clamping provides precise and complete electrophysiological information on ion channel function. Although conventional manual patch clamp (MPC) techniques have low throughput and are therefore not suitable for screening phase, automated patch clamp (APC) techniques are available and can be applied to investigate CFTR pharmacology.

Parallel planar APC provides a "medium throughput screening" technique with higher resolution than other screening methods and provides quantitative information on the electrophysiological properties of ion channels more rapidly than MPC (Farre et al., 2009). APC has become the gold standard for early safety assessment when examining cardiac hERG channel to new drug candidates (Polonchuk, 2012) but has not yet been widely used for CFTR pharmacology studies (Liu et al., 2009; Jacobsen and Sørensen, 2016; Brüggemann et al., 2017).

The objective of this study is to describe the application of APC technology to CFTR pharmacology. We recorded wt- and F508del-CFTR currents using planar APC under various conditions and compared the results with those obtained with MPC method.

\section{MATERIALS AND METHODS}

\section{Cell Culture}

Baby Hamster Kidney (BHK) cells were cultured in DMEM/F12 medium supplemented with 5\% FBS and 1\% Penicillin/Streptomycin. To study CFTR current, BHK were stably transfected with pNUT-wt-CFTR or pNUT-F508delCFTR plasmids using JetPeI reagent (Polyplus, Illkirch, France) according to the manufacturer's instructions. Clones were selected by addition of methotrexate $(500 \mu \mathrm{M})$ and CFTR expression validated by immunoblotting.

Chinese hamster ovary (CHO), HeLa, and CFBE41o [Cystic Fibrosis Bronchial Epithelial (CFBE)] cells were cultured as previously described (Kunzelmann et al., 1993; Grygorczyk et al., 1996; Hinzpeter et al., 2010). The CFBE cells developed in the laboratory of D. Gruenert had been transduced with wt- or F508del-CFTR containing the M470 polymorphism by Transzyme (Birmingham, AL, USA) and were kindly provided JP Clancy (Cincinnati Children's Hospital Medical Center).

Cells were cultured at $37^{\circ} \mathrm{C}$ in $5 \% \mathrm{CO}_{2}$ and were plated in $8.8 \mathrm{~cm}^{2}$ dishes for MPC and $21.6 \mathrm{~cm}^{2}$ dishes for APC. Patchclamp experiments were performed $2-3$ days after plating. APC needs cells in suspension, therefore, cells at $70-80 \%$ confluence were detached by incubation with Accutase (EMD Millipore, Billerica, MA, USA) for $5 \mathrm{~min}$ at $37^{\circ} \mathrm{C}$, suspended in complete culture, centrifuged for $2 \mathrm{~min}$ at $1000 \mathrm{rpm}$, and resuspended in DMEM/F12 without serum. Prior to use, the suspension was placed at $37^{\circ} \mathrm{C}$ for $15-20 \mathrm{~min}$ to allow cells to recover from the detachment process.

\section{Patch Clamp Experiments}

For conventional $\mathrm{MPC}, \mathrm{Cl}^{-}$currents were measured in the whole-cell patch-clamp configuration after compensating electronically for pipette capacitance in the cell-attached mode. Voltage-clamp signals were recorded using an Axopatch 200B amplifier connected to an analog/digital interface, Digidata $1440 \mathrm{~A}$, and were analyzed using pCLAMP 9 software (all from Axon Instruments Inc., Burlingame, CA, USA). Pipettes were pulled from borosilicate glass capillaries (GC150-TF10; Clark Electromedical Inc., Reading, UK) and connected to the head stage of the patch clamp amplifier through an $\mathrm{Ag}-\mathrm{AgCl}$ wire. The resistance of pipettes in the bath solution ranged between 4 and $6 \mathrm{M} \Omega$.

Automated whole-cell patch clamp recordings were obtained using two different systems. Most experiments were performed on the eight-channel Patchliner ${ }^{\circledR}$ NPC-16 workstation (Nanion Technologies $\mathrm{GmbH}$, Munich, Germany), which was coupled to two QuadroEPC-10 amplifiers (HEKA Elektronik GmbH, 
Germany). APC procedures followed Nanion's standard procedures and used Nanion's high-resistance chips. Some experiments were also performed using the Qpatch ${ }^{\circledR} 16 \mathrm{X}$ system (Sophion Bioscience, Ballerup, Denmark) with single-hole Qplates. Both chip models exhibited a resistance of 3-3.5 M $\Omega$.

Similar experiments were performed using APC and MPC. The holding potential was maintained at $-40 \mathrm{mV}$ throughout the experiment, and two voltage-clamp protocols were used to measure whole-cell CFTR currents. Figure 1 shows the sequence of events (called tree) used on the Nanion software (Figure 1A) and protocols applied for CFTR recording (Figure 1B). The tree of events shows all steps leading to the whole-cell configuration (gray rectangle 1) followed by the CFTR recording parameters (gray rectangle 2$)$. The current-voltage $(I-V)$ relationship $(I / V$ protocol, blue dots) was determined by pulsing from the holding potential of $-40 \mathrm{mV}$ to test potentials between -80 and $+80 \mathrm{mV}$ increasing in $20 \mathrm{mV}$ increments. To monitor the current evolution under drugs application and confirm the absence of significant leak current; a single depolarization from -40 to $0 \mathrm{mV}$ was applied every $5 \mathrm{~s}$ for 6-7 min (drug time course protocol, green dots).

\section{Medium and Chemicals}

Similar external and internal solutions were used for MPC and APC. The external bath solution contained (in mM): $145 \mathrm{NaCl}$, $4 \mathrm{CsCl}, 1 \mathrm{CaCl}_{2}, 1 \mathrm{MgCl}_{2}, 10$ glucose, and $10 \mathrm{TES}$ titrated with $\mathrm{NaOH}$ to $\mathrm{pH}$ 7.4. The osmolarity of the bath solution was $315 \pm 5 \mathrm{mOsmol}$. The intrapipette solution contained (in $\mathrm{mM}$ ): $113 \mathrm{~L}$-aspartic acid, $113 \mathrm{CsOH}, 27 \mathrm{CsCl}, 1 \mathrm{NaCl}, 1$ $\mathrm{MgCl}_{2}, 1$ ethylene glycol tetraacetic acid (EGTA), 10 TES, and 3 MgATP titrated to $\mathrm{pH} 7.2$ with $\mathrm{CsOH}$. Osmolarity of the pipette solution was $285 \pm 5 \mathrm{mOsmol}$. To facilitate giga-ohm (G $\Omega$ ) seal obtaining with the Patchliner ${ }^{\circledR}$ system, the external solution was transiently replaced by a seal enhancer solution during the sealing procedures. The seal enhancer solution contained (in $\mathrm{mM}$ ): 80 $\mathrm{NaCl}, 3 \mathrm{KCl}, 10 \mathrm{MgCl}_{2}, 35 \mathrm{CaCl}_{2}$, and $10 \mathrm{Hepes}\left(\mathrm{Na}^{+}\right.$salt) titrated with $\mathrm{HCl}$ to $\mathrm{pH}$ 7.4. Osmolarity of the seal enhancer solution was $298 \pm 5 \mathrm{mOsmol}$. After stable sealing of the cell, the seal enhancer solution was replaced by the external solution before performing the whole-cell configuration and recordings. Recordings were performed at room temperature $\left(20-25^{\circ} \mathrm{C}\right)$ unless stated otherwise.

VX809 and VX770 were from Selleckchem (Houston, TX, USA) and CFTR inh -172 was from Calbiochem (San Diego, CA, USA). All other products were from Sigma-Aldrich (SaintQuentin-Fallavier, France). Stock solutions were prepared in dimethylsulfoxide.

\section{Analysis and Statistics}

Manual patch clamp results were analyzed with pCLAMP, version 9, software (Axon Instruments). APC results were analyzed with Patch MasterPro software (HEKA) for Patchliner ${ }^{\circledR}$ experiments

\section{A}

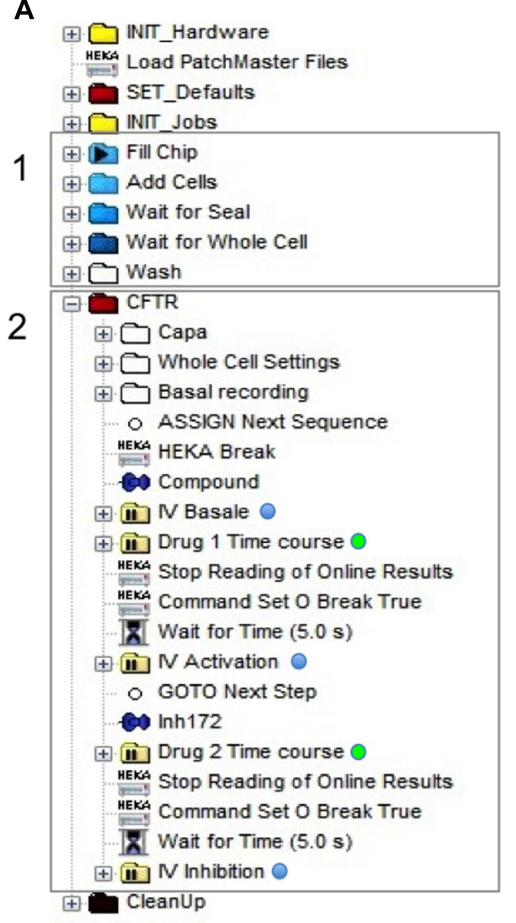

B

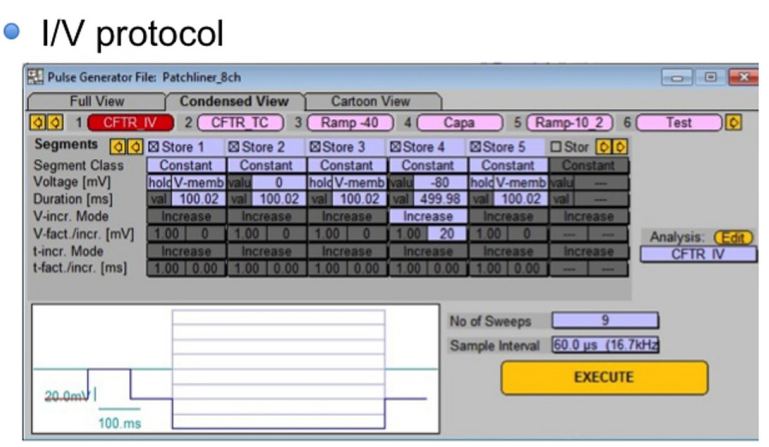

- Drug Time Course protocol

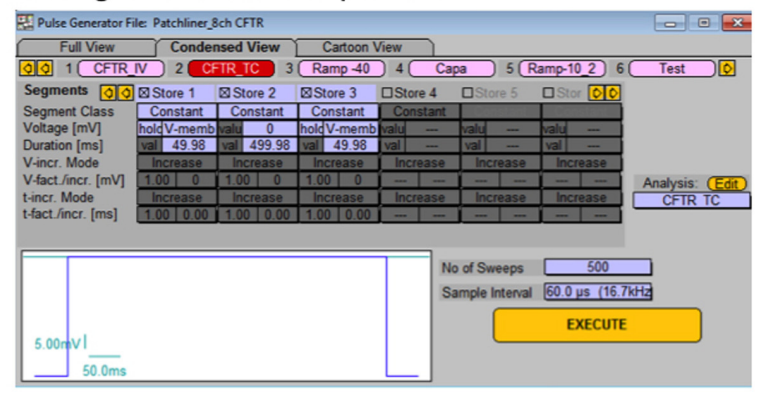

FIGURE 1 | Experimental procedure for automated patch clamp (APC) cystic fibrosis transmembrane conductance regulator (CFTR) recording. (A) Tree of events for whole-cell configuration establishing (gray rectangle 1) and personalized CFTR recording (gray rectangle 2). (B) The two different protocols applied for CFTR recording: IN protocol (blue dots) for current/voltage relationship visualization and drug time course (green dots) protocol to monitor the effect of compound in time. 
and with Sophion Analyzer Software (Sophion Bioscience) for Qpatch ${ }^{\circledR}$ experiments.

Results are expressed as means \pm SE of $n$ observations. Data were compared using the Student's $t$-test. Differences were considered statistically significant at $p<0.05$. All statistical tests were performed using GraphPad Prism, version 6.0 (GraphPad Software).

\section{RESULTS}

\section{Evaluation of APC Whole-cell Recording Conditions}

Automated planar patch clamp is commonly used to detect offtarget effects on the potassium channel hERG during the safety testing phase of drug development. Here, we describe its use for recording currents carried by wt-CFTR and rescued F508delCFTR channels and compare the results with those obtained from MPC recordings. In this study, we tested two APC devices: the Patchliner ${ }^{\circledR}$ from Nanion and the Qpatch ${ }^{\circledR}$ from Sophion Bioscience.

Both systems tested use the general principle of planar patch clamp, which is comparable to MPC with some differences in the experimental conditions (Milligan et al., 2009). Briefly, planar APC experiments use cartridge chips with a variable number of microfluidic chambers. Each chamber consists of two compartments containing extra- and intrasolutions, separated by a planar substrate (borosillicate glass for the Patchliner ${ }^{\circledR}$, siliconbased glass for the Qpatch ${ }^{\circledR}$ ) that has a micron-sized aperture. Cells in suspension are placed in the wells and interact with the glass to form stable giga-seal at the aperture. After gigaseal formation, whole-cell configuration is achieved by physical rupture of the cell-attached patch, then pre-programmed voltageclamp steps are applied and recording begins. Both systems used the microfluidic technology but with the following differences: Patchliner $^{\circledR}$ chips allow perfusion of external and internal solutions while Qpatch ${ }^{\circledR}$ has no internal perfusion. Another major difference is the higher throughput of the Qpatch ${ }^{\circledR}$, which possesses one or two arms, each with four channels, while the Patchliner ${ }^{\circledR}$ has only a single pipetting arm with one channel (see for review, Dunlop et al., 2008).

As APC requires both fresh cell suspension and cell membrane integrity, the first step was to obtain viable and isolated cells in suspension. We tested the $\mathrm{BHK}, \mathrm{CHO}, \mathrm{HeLa}$, and $\mathrm{CFBE}$ cell lines and several detachment protocols and reagents (e.g., TrypsinEDTA, Accutase). The best quality cells in suspension were obtained using Accutase and the detachment protocol detailed above in the Section "Materials and Methods." For all the tested cell lines, we achieved $90 \%$ cell viability (estimated by a manual counting of Trypan blue-stained cells) and well-separated cells without clusters.

Whole-cell-recording parameters and success rate for each cell line obtained with the Patchliner ${ }^{\circledR}$ system are shown in Table 1 along with the number of experiments that reached our criteria for a successful recording: a seal resistance of $1 \mathrm{G} \Omega\left(R_{\mathrm{m}}\right)$, wholecell access with a series resistance $<20 \mathrm{M} \Omega\left(R_{\mathrm{S}}\right)$, and a complete recording sequence (i.e., basal conditions - CFTR activation CFTR inhibition). Success rates varied between cell lines. The rates of achieving seals, entering the whole-cell configuration, and completing the CFTR recording protocol are shown in the table, with the overall success rates in pooled wt- and F508delCFTR experiments of 31.1 and $25.6 \%$ in $\mathrm{BHK}$ and $\mathrm{CHO}$ cell lines, respectively. Much lower rates were obtained in CFBE epithelial cells (5.2\%), although this might be improved with further optimization of the protocol. The following discussion focuses mainly on results obtained with BHK cells.

\section{Planar APC Recording of wt-CFTR}

Recordings were collected and analyzed in experiments that met the above criteria. In the following experiments, voltage pulse protocols were applied under basal conditions, in presence of the activator cocktail containing $10 \mu \mathrm{M}$ Forskolin (Fsk) $+30 \mu \mathrm{M}$ Genistein (Gst) and after further addition of $10 \mu \mathrm{M} \mathrm{CFTR}_{\text {inh }} 172$, a relatively selective CFTR inhibitor when added acutely (Ma et al., 2002). Briefly, Fsk regulates CFTR activity by stimulation of adenylate cyclase leading to the elevation of cAMP (Seamon and Daly, 1981) and PKA phosphorylation of the CFTR regulatory domain (R domain) (Tabcharani et al., 1991; Berger et al., 1993). The isoflavone Gst is a potent potentiator of the wt and F508delCFTR (Hwang et al., 1997).

Figure 2A shows representative whole-cell currents recorded in BHK-wt-CFTR cells using both Patchliner ${ }^{\circledR}$ automatic (left traces) and conventional (right traces) methods. Figure 2B shows the corresponding $I / V$ relationships. With the two experimental methods, Fsk + Gst elicited a robust $\mathrm{Cl}^{-}$current that was almost abolished by the specific inhibitor $\mathrm{CFTR}_{\text {inh }} 172$. However, the current density recorded by APC with Fsk + Gst was larger than the one recorded by MPC (respectively, at $+60 \mathrm{mV}$

TABLE 1 | Success rates of cystic fibrosis transmembrane conductance regulator (CFTR) recording with automated patch clamp (APC) experiments on Patchliner system.

\begin{tabular}{|c|c|c|c|c|c|}
\hline Cell line & Number of channel & $R_{\mathrm{m}}>1 \mathrm{G} \Omega$ & Whole cell $\left(R_{\mathrm{S}}<20 \mathrm{M} \Omega\right)$ & Recording & $\%$ Success \\
\hline Baby Hamster Kidney (BHK) & 87 & 60 & 51 & 27 & 31.1 \\
\hline Chinese hamster ovary $(\mathrm{CHO})$ & 86 & 48 & 39 & 22 & 25.6 \\
\hline HeLa & 133 & 71 & 57 & 18 & 13.5 \\
\hline Cystic Fibrosis Bronchial Epithelial (CFBE) & 38 & 5 & 3 & 2 & 5.2 \\
\hline
\end{tabular}

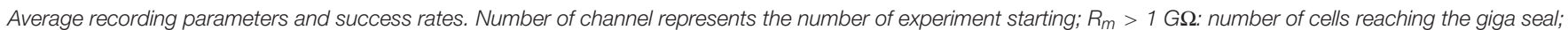

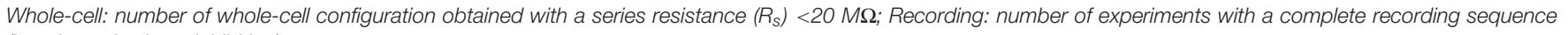
(basal - activation - inhibition). 


\section{A Automated Planar Patch Clamp}

\section{Basal}

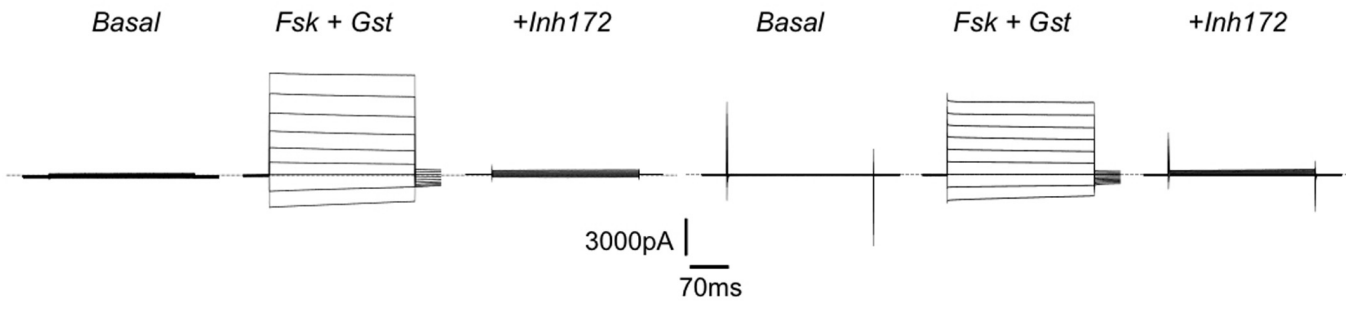

$+\operatorname{lnh} 172$

Basal

\section{Manual Patch Clamp}

B
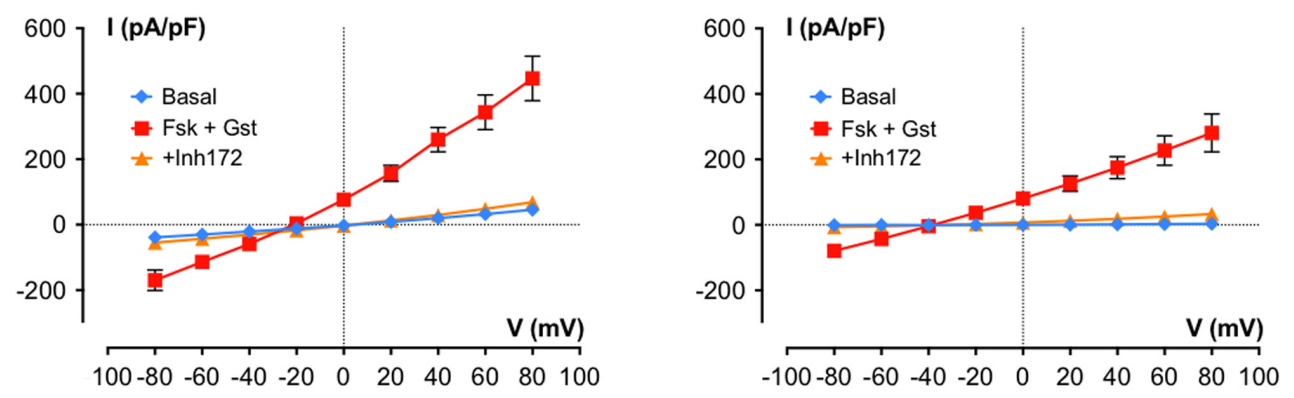

C

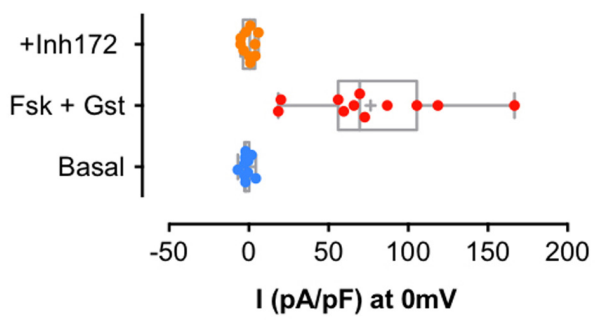

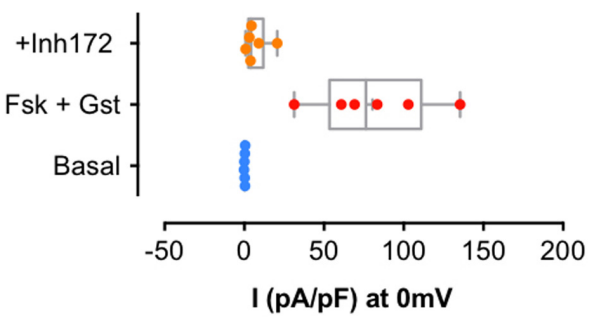

FIGURE 2 | Cystic fibrosis transmembrane conductance regulator whole-cell recording by automated and manual patch clamp from BHK-wt-CFTR. (A) Representative traces of whole-cell $\mathrm{Cl}^{-}$currents recorded by APC (left traces) or manual patch clamp (MPC; right traces) by stepping from a holding potential of $-40 \mathrm{mV}$ to a series of test potentials from -80 to $+80 \mathrm{mV}$ in $20 \mathrm{mV}$ increments under basal (unstimulated) conditions or in the presence of $10 \mu \mathrm{M}$ Fsk $+30 \mu \mathrm{M}$ Gst or $10 \mu \mathrm{M}$ CFTR $I_{\operatorname{lnh}} 172$ as indicated. Dashed lines indicate the zero current level. (B) Corresponding current densities (pA/pF) obtained by current-voltage relationships normalized by cell capacitance for APC (left traces; $n=11$ ) and MPC (right traces; $n=6$ ). (C) Corresponding distribution of current densities recorded at $0 \mathrm{mV}$. Error bars, SEM.

$343.4 \pm 52.8 \mathrm{pA} / \mathrm{pF} n=11$ and $227.4 \pm 45.5 \mathrm{pA} / \mathrm{pF} n=6$; $p=0.16)$ and displayed slight rectification and a reversal potential shift to $-20 \mathrm{mV}$ from the theoretical $E_{\mathrm{Cl}^{-}}$, which is expected to be near $-40 \mathrm{mV}$. Several observations suggest that the differences observed between data obtained using APC and MPC were caused by a decrease in the seal resistance while recording.

First, there were no differences when we compared the cell capacitance $\left(C_{\mathrm{m}}\right)$ and series resistance obtained with each method ( $p=0.46$ for $C_{\mathrm{m}}$ and $p=0.09$ for $R_{\mathrm{s}}$; Table 2) suggesting the current density obtained with APC was not increased artificially due to underestimation of the cell capacitance or reduced access resistance to the inside of the cell. Comparing the current densities at $+60 \mathrm{mV}$ under control conditions revealed a significant difference between basal currents measured under $\mathrm{APC}$ and MPC conditions $(32.3 \pm 6.3 \mathrm{pA} / \mathrm{pF} n=11$ and $2.4 \pm 0.4 \mathrm{pA} / \mathrm{pF}, n=6, p=0.003$, respectively) and basal conductance was not sensitive to $\mathrm{CFTR}_{\text {inh }} 172$ suggesting it was not mediated by CFTR (data not shown). By contrast, at $0 \mathrm{mV}$ (where the non-selective leak current would be undetectable),

TABLE 2 | Comparison of patch clamp parameters for manual and automated BHK whole-cell recordings.

\begin{tabular}{|c|c|c|c|c|}
\hline & \multicolumn{2}{|c|}{$\begin{array}{l}\text { Automated patch clamp } \\
\text { (APC) }\end{array}$} & \multicolumn{2}{|c|}{$\begin{array}{l}\text { Manual patch clamp } \\
\text { (MPC) }\end{array}$} \\
\hline & $C_{m}(p F)$ & $R_{\mathrm{S}}(\mathrm{M} \Omega)$ & $C_{m}(p F)$ & $R_{\mathrm{S}}(\mathrm{M} \Omega)$ \\
\hline BHK-wt-CFTR & $17.6 \pm 5.0$ & $18.9 \pm 2.5$ & $23.1 \pm 2.8$ & $12.4 \pm 1.4$ \\
\hline BHK-F508del-CFTR & $16.4 \pm 2.7$ & $10.3 \pm 1.3$ & $17.9 \pm 1.6$ & $13.0 \pm 1.4$ \\
\hline
\end{tabular}


A

\section{Automated Planar Patch Clamp}
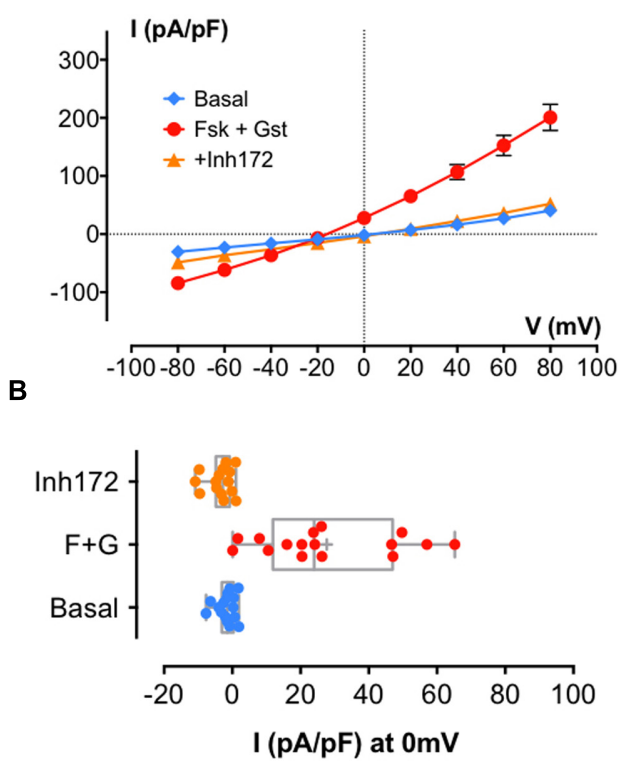

Manual Patch Clamp
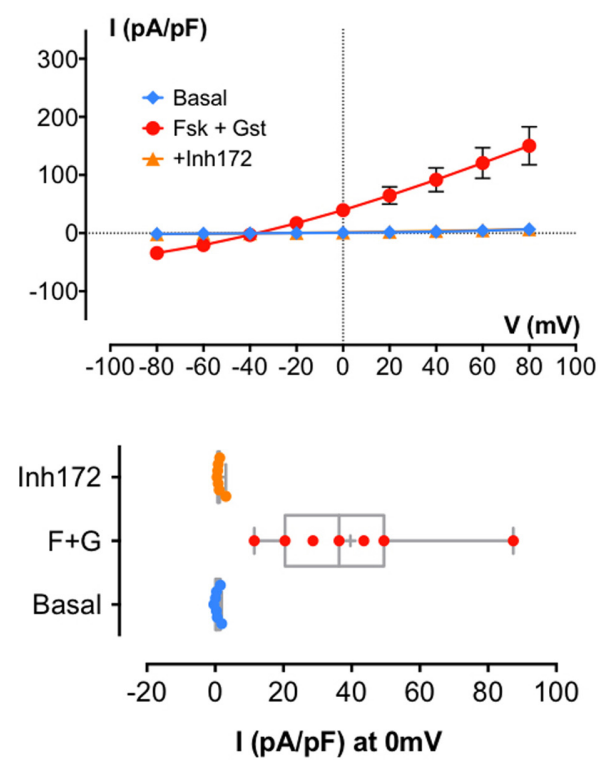

FIGURE 3 | Cystic fibrosis transmembrane conductance regulator whole-cell recording by automated and MPC from corrected (VX809 $24 \mathrm{~h}$ ) BHK-F508del-CFTR. (A) Current densities (pA/pF) obtained by current-voltage relationships normalized by cell capacitance on BHK-F508del-CFTR corrected $24 \mathrm{~h}$ with $10 \mu \mathrm{M} \mathrm{VX809}$. Whole-cell $\mathrm{Cl}^{-}$currents are recorded by APC (left traces; $n=16$ ) or MPC (right traces; $n=7$ ) by stepping from holding potential of $-40 \mathrm{mV}$ to a series of test potentials from -80 to $+80 \mathrm{mV}$ in $20 \mathrm{mV}$ increments under basal (unstimulated) conditions or in the presence of $10 \mu \mathrm{M} \mathrm{Fsk}+30 \mu \mathrm{M}$ Gst or $10 \mu \mathrm{M}$ CFTRIIn 172 as indicated. (B) Corresponding distribution of current densities recorded at $0 \mathrm{mV}$. Error bars, SEM.

current densities recorded in the presence of Fsk + Gst were similar for both methods $(76.4 \pm 12.9 \mathrm{pA} / \mathrm{pF}, n=11$ with APC and $80.5 \pm 14.7, n=6$ with MPC; n.s. $p=0.85$; Figure $2 \mathrm{C}$ ).

\section{APC Recording of Corrected F508del-CFTR}

F508del is by far the most common CF mutation; therefore, the functional screening assay should enable the recording of F508del-CFTR currents. However, this mutation induces a gating defect in addition to the trafficking defect (Dalemans et al., 1991); therefore, the assay should be sensitive enough to accurately record potentiator-induced increases in $\mathrm{Cl}^{-}$current so that different potentiator and corrector drugs can be compared. In another set of experiments, we compared F508del-CFTR current recordings obtained with the APC (Patchliner ${ }^{\circledR}$ ) and MPC methods.

First, we used BHK-F508del-CFTR cells under control conditions (DMSO treatment for $24 \mathrm{~h}$ ). As expected, wholecell recording detected little for any $\mathrm{Cl}^{-}$current using either method (current densities at $0 \mathrm{mV}:-0.67 \pm 1.1 \mathrm{pA} / \mathrm{pF}, n=10$ with $\mathrm{APC}$ and $6.9 \pm 2.9 \mathrm{pA} / \mathrm{pF}, n=6$ with MPC; data not shown). When the same experiments were performed after a 24 -h pretreatment with the corrector VX809 $(10 \mu \mathrm{M})$, CFTR current was detected. Figure 3A shows $I / V$ relationships obtained using APC (left curves) and MPC (right curves) under basal conditions and during CFTR activation and inhibition. Figure $3 \mathbf{B}$ shows the corresponding distribution of each current density at $0 \mathrm{mV}$. As with wt-CFTR, Fsk + Gst elicited large F508del-CFTR currents that were nearly abolished by $\mathrm{CFTR}_{\text {inh }} 172$ and, as previously observed, the APC current densities were somewhat larger and the reversal potential were shifted compared to MPC. Despite these differences, electrophysiological parameters were not significantly different (Table $2 ; p=0.73$ for $C_{\mathrm{m}}$ and $p=0.24$ for $R_{\mathrm{S}}$ ) nor were the current densities at $0 \mathrm{mV}$ (Figure 3B; $27.70 \pm 4.9 \mathrm{pA} / \mathrm{pF}, n=16$ for $\mathrm{APC}$ and $39.62 \pm 9.3 \mathrm{pA} / \mathrm{pF}$, $n=7$ for MPC, $p=0.23$ ). Taken together, these results indicate that, aside from a slight contamination by leak current with APC, similar results can be obtained when recording rescued F508del-CFTR using either method.

\section{Variety of Recording Applications}

The recording sequence above is useful when determining the efficiency of candidate corrector molecules or comparing the potencies of several correctors. However, with MPC, we are potentially able to collect information such as the stability of currents over long time periods and the temperature dependence of channel gating. We examined the capabilities of APC for such experiments with Patchliner ${ }^{\circledR}$ system (Figure 4).

To study the stability of whole-cell currents, we performed APC whole-cell recordings on BHK cells over a 20-min period. Figure $4 \mathrm{~A}$ shows the mean of wt-CFTR $\mathrm{Cl}^{-}$current time courses in the presence of Fsk + Gst $(n=5)$. For these experiments, 

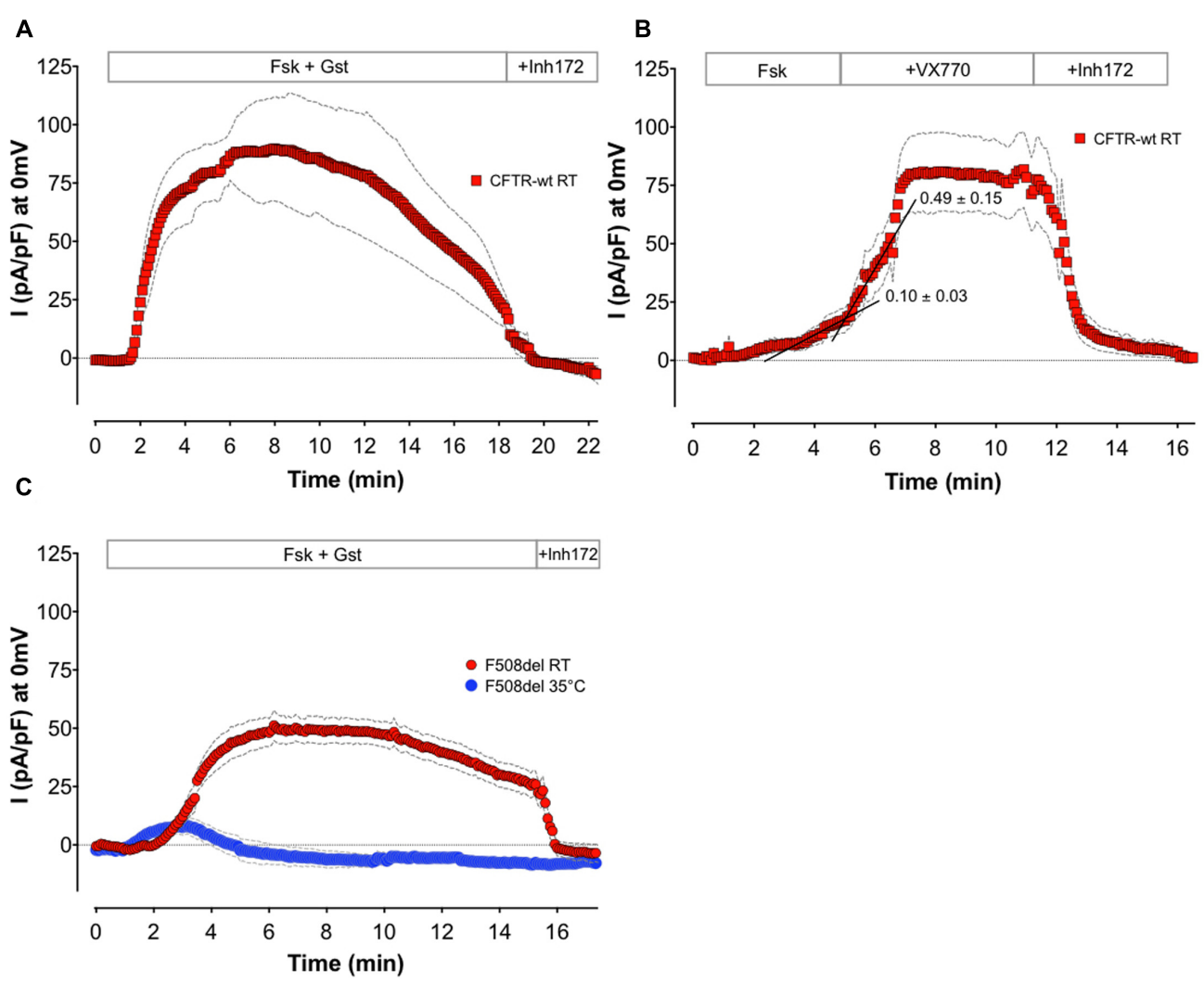

FIGURE 4 | Alternative application recorded with APC. Means of whole-cell $\mathrm{Cl}^{-}$current density time courses at $0 \mathrm{mV}$ recorded on $\mathrm{BHK}$-wt-CFTR (A,B) or BHK-F508del-CFTR (C) by APC. (A) Monitoring of wt channels rundown by long recording in presence of $10 \mu \mathrm{M}$ Fsk $+30 \mu \mathrm{M}$ Gst $(n=5)$. (B) Monitoring CFTR potentiation by sequential addition of $1 \mu \mathrm{M}$ Fsk, and then $10 \mu \mathrm{M} \mathrm{VX770}(n=8)$. Black lines are representative linear regressions of the time course curves before and after potentiator addition. Means of slope values are indicated. (C) Effect of temperature on F508del channel function: recording of whole-cell current at room (RT; $n=6)$ or physiological temperature $\left(35^{\circ} \mathrm{C} ; n=5\right)$ in presence of $10 \mu \mathrm{M}$ Fsk $+30 \mu \mathrm{M}$ Gst. For all conditions, $10 \mu \mathrm{M}$ CFTR inh $_{172}$ was added at the end of experiment. Colored symbols are the mean of current densities and gray dashed lines the corresponding error, SEM.

Rs were determined at the beginning and end of each recording and did not vary more than $25 \%$ from the initial value. As for MPC, we can record long enough with APC to observe the physiological rundown of CFTR channels (Becq et al., 1994). Such an experiment may be considered when assaying the ability of compounds to increase CFTR stability. In addition, the high quality of the seal and stability of the patch may allow the effects of sequential drug applications to be monitored and determination of $\mathrm{EC}_{50}$ or $\mathrm{IC}_{50}$. Figure $4 \mathrm{~B}$ shows the mean of wtCFTR current time courses at $0 \mathrm{mV}$ with sequential addition of sub-maximal Fsk $(1 \mu \mathrm{M})$ followed by $10 \mu \mathrm{M} \operatorname{VX770}(n=8)$. To evaluate the potentiator effect of the tested compound, linear regression analyses were performed using the points on each time course curve. For each individual experiment, points during the $2 \mathrm{~min}$ before and the $2 \mathrm{~min}$ after compound addition were used to calculate the slopes. The example in Figure 4B (black lines) shows representative linear regression before and after addition of VX770. Here, the mean slopes computed for eight different cells yielded values that are significantly different $(0.101 \pm 0.031$ for Fsk alone and $0.499 \pm 0.149$ after $10 \mu \mathrm{M}$ VX770 addition, $n=8, p=0.02$ ) and clearly demonstrate potentiation of the Fsk-activated current by the potentiator VX770.
Finally, the temperature dependence of F508del CFTR function was examined using APC. Whole-cell recordings were obtained at room temperature and at $35^{\circ} \mathrm{C}$ using BHK-F508delCFTR cells that had been pretreated for $24 \mathrm{~h}$ with $10 \mu \mathrm{M}$ VX809. Figure 4C shows the mean of current densities at $0 \mathrm{mV}$ during stimulation of F508del-CFTR by Fsk + Gst at $22^{\circ} \mathrm{C}(n=6)$ or $35^{\circ} \mathrm{C}(n=5)$. According to the literature (Wang et al., 2011, 2014; Liu et al., 2012) and contrary to the CFTR response obtained at $22^{\circ} \mathrm{C}$, only a small transient current is recorded at physiological temperature. These results show that whole-cell CFTR currents can be studied at $37^{\circ} \mathrm{C}$ using APC, allowing drug effects to be studied under physiological conditions. Also, it suggests the feasibility of screening for compounds that correct the thermal instability of F508delCFTR.

\section{Planar APC Using Other Cells and Robotic Systems}

The experiments described above were performed using BHK cells and the Patchliner ${ }^{\circledR}$ APC system. However, as mentioned in Table 1, we also tested APC with different cell types. Figures 5A,B 


\section{A}

MPC recording of CHO-CFTR-wt

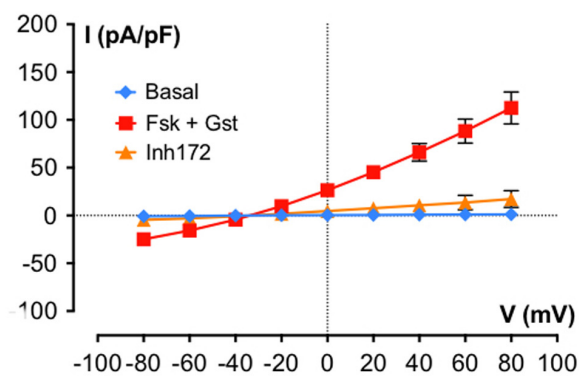

C

Qpatch® recording of CHO-CFTR-wt

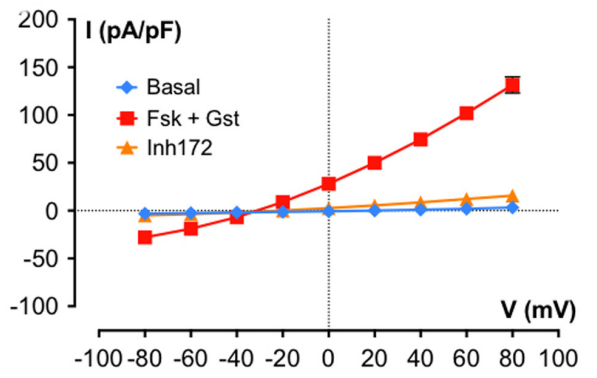

B

Patchliner® recording of $\mathrm{CHO}-\mathrm{CFTR}$ -

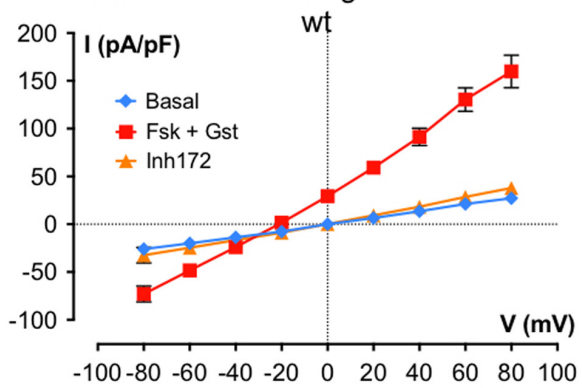

D

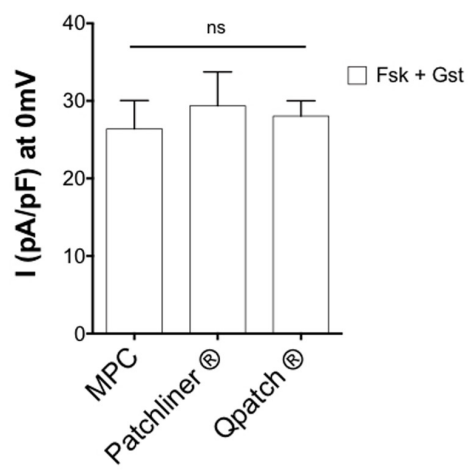

FIGURE 5 | Cystic fibrosis transmembrane conductance regulator whole-cell recording by automated and MPC from Chinese hamster ovary (CHO) cells stably expressing wt-CFTR. (A-C) Current densities (pA/pF) obtained by current-voltage relationships normalized by cell capacitance on CHO-wt-CFTR. Whole-cell $\mathrm{Cl}^{-}$currents are recorded with MPC $(\mathbf{A}, n=4)$ or with APC with two workstations: the Nanion Patchliner $(\mathbf{B}, n=10)$ or the Sophion Qpatch $(\mathbf{C}, n=60)$ by stepping from a holding potential of $-40 \mathrm{mV}$ to a series of test potentials from -80 to $+80 \mathrm{mV}$ in $20 \mathrm{mV}$ increments under basal (unstimulated) conditions or in the presence of $10 \mu \mathrm{M}$ Fsk $+30 \mu \mathrm{M}$ Gst or $10 \mu \mathrm{M}$ CFTRInh 172 as indicated. (D) Histograms of current densities recorded at $0 \mathrm{mV}$ in the presence of Fsk + Gst. Error bars, SEM; ns, not significant.

show $I / V$ relationships obtained using MPC or APC to study $\mathrm{CHO}$ cells stably expressing wt-CFTR. Cells were recorded under basal conditions and in the presence of $10 \mu \mathrm{M}$ Fsk $+30 \mu \mathrm{M}$ Gst and $10 \mu \mathrm{M} \mathrm{CFTR}_{\text {Inh }} 172$. With both methods, the activator cocktail Fsk + Gst, elicited strong voltage- and time-independent currents that were nearly abolished by $\mathrm{CFTR}_{\text {inh }} 172$. A small shift in the reversal potential was observed with APC $(-20 \mathrm{mV}$ instead of the expected $-40 \mathrm{mV}$ ); nevertheless, robust CFTR recordings were obtained with $\mathrm{CHO}$ cells stably expressing wtCFTR, which resembled those obtained with BHK except for having lower current densities as expected with lower CFTR expression.

Finally, we tested CFTR recording using another robotic APC system: the Sophion QPatch ${ }^{\circledR}$. Using QPatch ${ }^{\circledR}$, the rates of success obtained were better for CHO-wt-CFTR cells, and the CFTR whole-cell recordings made with the same protocols and medium were close to those obtained previously (Figure 5C).

In summary, detailed comparison of the current densities at $0 \mathrm{mV}$ using the MPC or two APC systems suggests they yield very similar results (Figure $5 \mathrm{D} ; I(\mathrm{pA} / \mathrm{pF}$ ) at $0 \mathrm{mV}: 29.37 \pm 4.4 \mathrm{pA} / \mathrm{pF}$, $n=10$ for the Patchliner ${ }^{\otimes} ; 28.04 \pm 1.9 \mathrm{pA} / \mathrm{pF}, n=60$ for Qpatch $^{\circledR}$ and $26.40 \pm 3.6 \mathrm{pA} / \mathrm{pF}, n=4$ for MPC) and provide good reproducibility between different APC systems and cell models.

\section{DISCUSSION}

Several cell-based screening assays for CFTR are available that monitor anion flux [e.g., using radiotracer efflux (Venglarik et al., 1990) or YFP-based halide transport (Galietta et al., 2001)]. Although they provide high throughput, they are only semi-quantitative because the net electrochemical across the membrane for the anion changes during the course of the assay. Here, we have shown that planar APC can be used when screening for CFTR modulators and provides medium throughput together with several advantages over other cellbased assays.

APC allows wt- and corrected F508del-CFTR activity to be recorded with high efficiency and resolution approaching that of MPC, but at much faster rates. Optimizing the success rate is key to using these assays for drug testing. In the present study, success rates reached $31 \%$ for $\mathrm{BHK}$ and $25.6 \%$ for $\mathrm{CHO}$ cells, which are acceptable though values lower than that were found in the literature for similar cell lines and the same APC system (Milligan et al., 2009). To increase this rate of experiment success and develop the use of more physiological cell lines (i.e., CFBE and Calu-3), the cell preparation needs to be improved, e.g., by adapting protocols for culturing adherent cell lines in suspension (Muller et al., 2005). 
CFTR recordings obtained using APC are similar to those obtained using MPC: no CFTR current was detected under basal conditions but it was strongly activated by Fsk + Gst and abolished by the specific CFTR inhibitor CFTR $_{\text {inh }} 172$. The main difference was that CFTR currents recorded using APC were often contaminated with non-selective leak currents due to a decline in seal quality. Since the user can't easily intervene during the APC experiments, they require controls and welldesigned protocols to allow the discrimination of false positives. In our experiments, we replaced potassium ions with cesium to block $\mathrm{K}^{+}$currents and designed patch solutions with an $E_{\mathrm{Cl}^{-}}$ far from $0 \mathrm{mV}$ so that leak and CFTR currents could be easily discriminated.

Interestingly, APC can use the same protocols as MPC, making it useful for studies of current stability over long periods, the effects of sequential drug applications (useful for $\mathrm{EC}_{50}$ or $\mathrm{IC}_{50}$ determination, precise measurement of potentiation), and the temperature dependence of channel gating CFTR modulators. This last point is a real advantage compared to many HTS assay since it enables compounds to be tested at physiological temperature. As already mentioned, mutant CFTR channels have altered temperature sensitivity, yet, most screening assays are performed at room temperature, which may explain some discrepancies between in vitro results and clinical trials. Similar results were obtained using the two APC systems suggesting either planar APC methods can be used for CFTR studies.

Once the conditions have been optimized, APC is faster and easier to use than MPC with similar high-resolution results and the possibility to address the same questions. All steps during APC experiments are finely controlled and executed by a robot, allowing more consistent times and concentrations at which drugs are injected. The use of microfluidics within the APC chips ensures that volumes are minimized and allows more precise control of the temperature.

A major drawback with APC is the requirement for cells in suspension, which limits the use of epithelial cell lines under physiological conditions, including CFBE, Calu-3, and the human colonic cell line T84. The normal state of these cells

\section{REFERENCES}

Becq, F., Jensen, T. J., Chang, X. B., Savoia, A., Rommens, J. M., Tsui, L. C., et al. (1994). Phosphatase inhibitors activate normal and defective CFTR chloride channels. Proc. Natl. Acad. Sci. U.S.A. 91, 9160-9164. doi: 10.1073/pnas.91.19. 9160

Berger, H. A., Travis, S. M., and Welsh, M. J. (1993). Regulation of the cystic fibrosis transmembrane conductance regulator $\mathrm{Cl}$ - channel by specific protein kinases and protein phosphatases. J. Biol. Chem. 268, 2037-2047.

Boyle, M. P., Bell, S. C., Konstan, M. W., McColley, S. A., Rowe, S. M., Rietschel, E., et al. (2014). A CFTR corrector (lumacaftor) and a CFTR potentiator (ivacaftor) for treatment of patients with cystic fibrosis who have a phe508del CFTR mutation: a phase 2 randomised controlled trial. Lancet Respir. Med. 2, 527-538. doi: 10.1016/S2213-2600(14)70132-8

Brüggemann, A., Friis, S., Strassmeier, T., Rapedius, M., Goetze, T., Rinke, I., et al. (2017). Characterization of CFTR activators and inhibitors by the use of a planar patch clamp system. Biophys. J. 112, 411a. doi: 10.1016/j.bpj.2016.11.2561

Cheng, S. H., Gregory, R. J., Marshall, J., Paul, S., Souza, D. W., White, G. A., et al. (1990). Defective intracellular transport and processing of CFTR is the is highly polarized, with tight junctions and apical CFTR. The effects of CFTR modulators may be influenced by the integrity of the epithelium and the maintenance of cell-cell contacts (Fritsch and Edelman, 1996). The detachment protocol used in this study or the generation of cell lines adapted to suspension, may also adversely affect interactions between CFTR and its partners, altering channel function and regulation.

\section{CONCLUSION}

We have shown that whole-cell parallel planar APC is suitable for recording CFTR activity and gives robust results similar to those obtained by MPC but with higher throughput and standardized protocols. Screening with APC is an attractive option for pharmacological investigations including hit validation, trafficking correction, potentiation, thermal stability restoration, and the functional characterization of mutant CFTR.

\section{AUTHOR CONTRIBUTIONS}

$\mathrm{AB}, \mathrm{LF}, \mathrm{JH}$, and $\mathrm{FB}$ designed the experiments. $\mathrm{AB}$ and $\mathrm{LF}$ performed experiments. $\mathrm{AB}$ and $\mathrm{LF}$ analyzed the data. $\mathrm{AB}, \mathrm{LF}, \mathrm{JH}$, and FB wrote the manuscript.

\section{FUNDING}

This work was funded by the Cystic Fibrosis Trust (SRC 005, to $\mathrm{AB}$ and $\mathrm{FB}$ ), the association Vaincre la mucoviscidose (to $\mathrm{FB}$ and $\mathrm{LF}$ ), the CPER/FEDER and région Poitou-Charentes (to FB), and the Canadian Institutes of Health Research and Cystic Fibrosis Canada (to JH).

\section{ACKNOWLEDGMENT}

The authors thank S. Mirval for technical assistance.

molecular basis of most cystic fibrosis. Cell 63, 827-834. doi: 10.1016/00928674(90)90148-8

Dalemans, W., Barbry, P., Champigny, G., Jallat, S., Dott, K., Dreyer, D., et al. (1991). Altered chloride ion channel kinetics associated with the delta F508 cystic fibrosis mutation. Nature 354, 526-528. doi: 10.1038/354526a0

Dunlop, J., Bowlby, M., Peri, R., Vasilyev, D., and Arias, R. (2008). Highthroughput electrophysiology: an emerging paradigm for ion-channel screening and physiology. Nat. Rev. Drug Discov. 7, 358-368. doi: 10.1038/nrd 2552

Farre, C., Haythornthwaite, A., Haarmann, C., Stoelzle, S., Kreir, M., George, M., et al. (2009). Port-a-patch and patchliner: high fidelity electrophysiology for secondary screening and safety pharmacology. Comb. Chem. High Throughput Screen. 12, 24-37. doi: 10.2174/138620709787047966

Fritsch, J., and Edelman, A. (1996). Modulation of the hyperpolarization-activated Cl- current in human intestinal T84 epithelial cells by phosphorylation. J. Physiol. 490, 115-128. doi: 10.1113/jphysiol.1996.sp021130

Galietta, L. V., Jayaraman, S., and Verkman, A. S. (2001). Cell-based assay for highthroughput quantitative screening of CFTR chloride transport agonists. Am. J. Physiol. Cell Physiol. 281, C1734-C1742. 
Grygorczyk, R., Tabcharani, J. A., and Hanrahan, J. W. (1996). CFTR channels expressed in $\mathrm{CHO}$ cells do not have detectable ATP conductance. J. Membr. Biol. 151, 139-148. doi: 10.1007/s002329900065

Hamill, O. P., Marty, A., Neher, E., Sakmann, B., and Sigworth, F. J. (1981). Improved patch-clamp techniques for high-resolution current recording from cells and cell-free membrane patches. Pflugers Arch. 391, 85-100. doi: 10.1007/ BF00656997

Hinzpeter, A., Aissat, A., Sondo, E., Costa, C., Arous, N., Gameiro, C., et al. (2010). Alternative splicing at a NAGNAG acceptor site as a novel phenotype modifier. PLoS Genet. 6:e1001153. doi: 10.1371/journal.pgen. 1001153

Hwang, T. C., Wang, F., Yang, I. C., and Reenstra, W. W. (1997). Genistein potentiates wild-type and delta F508-CFTR channel activity. Am. J. Physiol. 273, C988-C998.

Jacobsen, R. B., and Sørensen, N. M. M. (2016). CFTR on an automated patch clamp system. Biophys. J. 110, 452a. doi: 10.1016/j.bpj.2015.11.2426

Kunzelmann, K., Schwiebert, E. M., Zeitlin, P. L., Kuo, W. L., Stanton, B. A., and Gruenert, D. C. (1993). An immortalized cystic fibrosis tracheal epithelial cell line homozygous for the delta F508 CFTR mutation. Am. J. Respir. Cell Mol. Biol. 8, 522-529. doi: 10.1165/ajrcmb/8.5.522

Liu, X., O’Donnell, N., Landstrom, A., Skach, W. R., and Dawson, D. C. (2012). Thermal instability of delf508 cystic fibrosis transmembrane conductance regulator (CFTR) channel function: protection by single suppressor mutations and inhibiting channel activity. Biochemistry 51, 5113-5124. doi: 10.1021/ bi300018e

Liu, Y., Mathes, C., Friis, S., and Finley, M. (2009). QPatch: the missing link between HTS and ion channel drug discovery. Comb. Chem. High Throughput Screen. 12, 78-95. doi: 10.2174/138620709787 047948

Lukacs, G. L., Chang, X. B., Bear, C., Kartner, N., Mohamed, A., Riordan, J. R., et al. (1993). The delta F508 mutation decreases the stability of cystic fibrosis transmembrane conductance regulator in the plasma membrane. Determination of functional half-lives on transfected cells. J. Biol. Chem. 268, 21592-21598.

Ma, T., Thiagarajah, J. R., Yang, H., Sonawane, N. D., Folli, C., and Galietta, L. J. V, et al. (2002). Thiazolidinone CFTR inhibitor identified by high-throughput screening blocks cholera toxin-induced intestinal fluid secretion. J. Clin. Invest. 110, 1651-1658. doi: 10.1172/JCI16112

Maitra, R., Sivashanmugam, P., and Warner, K. (2013). A rapid membrane potential assay to monitor CFTR function and inhibition. J. Biomol. Screen. 18, 1132-1137. doi: 10.1177/1087057113488420

Milligan, C. J., Li, J., Sukumar, P., Majeed, Y., Dallas, M. L., English, A., et al. (2009). Robotic multiwell planar patch-clamp for native and primary mammalian cells. Nat. Protoc. 4, 244-255. doi: 10.1038/nprot. 2008.230

Muller, N., Girard, P., Hacker, D. L., Jordan, M., and Wurm, F. M. (2005). Orbital shaker technology for the cultivation of mammalian cells in suspension. Biotechnol. Bioeng. 89, 400-406. doi: 10.1002/bit.20358
Pedemonte, N., Zegarra-Moran, O., and Galietta, L. J. (2011). High-throughput screening of libraries of compounds to identify CFTR modulators. Methods Mol. Biol. 741, 13-21. doi: 10.1007/978-1-61779-117-8_2

Polonchuk, L. (2012). Toward a new gold standard for early safety: automated temperature-controlled hERG test on the PatchLiner ${ }^{\circledR}$ Front. Pharmacol. 3:3. doi: 10.3389/fphar.2012.00003

Riordan, J. R., Rommens, J. M., Kerem, B., Alon, N., Rozmahel, R., Grzelczak, Z., et al. (1989). Identification of the cystic fibrosis gene: cloning and characterization of complementary DNA. Science 245, 1066-1073. doi: 10.1126/ science. 2475911

Seamon, K. B., and Daly, J. W. (1981). Forskolin: a unique diterpene activator of cyclic AMP-generating systems. J. Cyclic Nucleotide Res. 7, 201-224.

Tabcharani, J. A., Chang, X. B., Riordan, J. R., and Hanrahan, J. W. (1991). Phosphorylation-regulated Cl- channel in $\mathrm{CHO}$ cells stably expressing the cystic fibrosis gene. Nature 352, 628-631. doi: 10.1038/352628a0

Van Goor, F., Hadida, S., Grootenhuis, P. D. J., Burton, B., Cao, D., Neuberger, T., et al. (2009). Rescue of CF airway epithelial cell function in vitro by a CFTR potentiator, VX-770. Proc. Natl. Acad. Sci. U.S.A. 106, 18825-18830. doi: 10.1073/pnas.0904709106

Van Goor, F., Hadida, S., Grootenhuis, P. D. J., Burton, B., Stack, J. H., Straley, K. S., et al. (2011). Correction of the F508del-CFTR protein processing defect in vitro by the investigational drug VX-809. Proc. Natl. Acad. Sci. U.S.A. 108, 18843-18848. doi: 10.1073/pnas.1105787108

Van Goor, F., Straley, K. S., Cao, D., González, J., Hadida, S., Hazlewood, A., et al. (2006). Rescue of $\Delta$ F508-CFTR trafficking and gating in human cystic fibrosis airway primary cultures by small molecules. Am. J. Physiol. Lung Cell. Mol. Physiol. 290, L1117-L1130. doi: 10.1152/ajplung.00169.2005

Venglarik, C. J., Bridges, R. J., and Frizzell, R. A. (1990). A simple assay for agonistregulated $\mathrm{Cl}$ and $\mathrm{K}$ conductances in salt-secreting epithelial cells. Am. J. Physiol. 259, C358-C364.

Wang, W., Okeyo, G. O., Tao, B., Hong, J. S., and Kirk, K. L. (2011). Thermally unstable gating of the most common cystic fibrosis mutant channel ( $\Delta \mathrm{F} 508)$. J. Biol. Chem. 286, 41937-41948. doi: 10.1074/jbc.M111.296061

Wang, Y., Liu, J., Loizidou, A., Bugeja, L. A., Warner, R., Hawley, B. R., et al. (2014). CFTR potentiators partially restore channel function to A561E-CFTR, a cystic fibrosis mutant with a similar mechanism of dysfunction as F508del-CFTR. Br. J. Pharmacol. 171, 4490-4503. doi: 10.1111/bph.12791

Conflict of Interest Statement: The authors declare that the research was conducted in the absence of any commercial or financial relationships that could be construed as a potential conflict of interest.

Copyright (C) 2017 Billet, Froux, Hanrahan and Becq. This is an open-access article distributed under the terms of the Creative Commons Attribution License (CC BY). The use, distribution or reproduction in other forums is permitted, provided the original author(s) or licensor are credited and that the original publication in this journal is cited, in accordance with accepted academic practice. No use, distribution or reproduction is permitted which does not comply with these terms. 